\title{
STABILITY OF FUNCTIONAL EQUATIONS IN DISLOCATED QUASI-METRIC SPACES
}

\author{
Beata Hejmej
}

\begin{abstract}
We present a result on the generalized Hyers-Ulam stability of a functional equation in a single variable for functions that have values in a complete dislocated quasi-metric space. Next, we show how to apply it to prove stability of the Cauchy functional equation and the linear functional equation in two variables, also for functions taking values in a complete dislocated quasimetric space. In this way we generalize some earlier results proved for classical complete metric spaces.
\end{abstract}

\section{Introduction and preliminaries}

The stability of functional equations is a problem originating from the following question formulated by S.M. Ulam (see [5, 11]) for group homomorphisms:

Assume that $\left(G_{1},+\right)$ and $\left(G_{2},+\right)$ are groups, $\left(G_{2}, d\right)$ is a metric space and $\varepsilon>0$. Find $\delta>0$ such that for any function $\varphi: G_{1} \rightarrow G_{2}$ with

$$
d(\varphi(x+y), \varphi(x)+\varphi(y)) \leqslant \delta, x, y \in G_{1},
$$

there exists a group homomorphism $F: G_{1} \rightarrow G_{2}$ such that

$$
d(\varphi(x), F(x)) \leqslant \varepsilon, x \in G_{1} .
$$

Received: 30.09.2017. Accepted: 11.04.2018. Published online: 14.05.2018.

(2010) Mathematics Subject Classification: 39B82, 54E50.

Key words and phrases: stability of functional equations, square symmetric groupoid, dislocated quasi-metric space, semigroup, Cauchy equation. 
The first partial answer to it was published by D.H. Hyers [5] in 1941 and since that year, a lot of analogous results have been appearing (see, e.g., [3, 6]).

In this paper we consider a similar problem for so called dislocated quasimetrics (dq-metrics) instead of metrics. Namely, we show that 44, Theorem 2.1], proved for classical metric spaces, is valid also for dq-metric spaces. Moreover, we present some applications of that result, analogously as in [4].

Dislocated quasi-metrics play a crucial role, among others, in computer science and cryptography. Below we recall the definition of them.

Let $Y$ be a nonempty set with a function $d: Y \times Y \rightarrow[0,+\infty)$, which satisfies the following two conditions:

(Q1) $d(x, y)=d(y, x)=0 \Rightarrow x=y$,

(Q2) $d(x, y) \leqslant d(x, z)+d(z, y)$

for all $x, y, z \in Y$. In this case $d$ is said to be a dislocated quasi-metric ( $d q$ metric for short) and $(Y, d)$ is called a dislocated quasi-metric space (dq-metric space for short) (see [10, 9]). If $d$ is a dq-metric in $Y$, which is symmetric (i.e., $d(x, y)=d(y, x)$ for $x, y \in Y)$, then $(Y, d)$ is called a metric-like space (see [1]).

Example $1.1([9])$. A function $d: \mathbb{R} \times \mathbb{R} \rightarrow[0, \infty)$ defined by $d(x, y)=|x|$ for $x, y \in \mathbb{R}$ is a dislocated quasi-metric.

EXAmple $1.2([\mathbb{1})$. If $d: \mathbb{R} \times \mathbb{R} \rightarrow[0, \infty)$ is given by $d(x, y)=\max \{|x|,|y|\}$ for $x, y \in \mathbb{R}$, then $(\mathbb{R}, d)$ is a metric-like space.

We say that $x \in Y$ is a limit of a sequence $\left(x_{n}\right)_{n=1}^{\infty}$ of elements in a dqmetric space $(Y, d)$ iff $d\left(x_{n}, x\right) \rightarrow 0$ and $d\left(x, x_{n}\right) \rightarrow 0$; we then write $x_{n} \rightarrow x$. It is easy to check that a limit of a sequence is unique if it exists. Indeed, if $x, y \in Y$ are limits of a sequence $\left(x_{n}\right)_{n=1}^{\infty}$, then for all $n \in \mathbb{N}$ we have $0 \leqslant d(x, y) \leqslant d\left(x, x_{n}\right)+d\left(x_{n}, y\right)$. Thus, $d(x, y)=0$ and similarly, $d(y, x)=0$, so $x=y$.

A sequence $\left(x_{n}\right)_{n=1}^{\infty}$ is said to be a Cauchy sequence if

$$
\forall_{\varepsilon>0} \exists_{N \in \mathbb{N}} \forall_{m, n \geqslant N} d\left(x_{n}, x_{m}\right)<\varepsilon .
$$

A dq-metric space $(Y, d)$ is called complete if every Cauchy sequence has a limit in this space. 


\section{Main theorem}

Let $(Y, d)$ be a complete dq-metric space. Consider a nonempty set $K$ and functions $\Psi: Y \rightarrow Y, \alpha: K \rightarrow K$, and $h_{1}, h_{2}: K \rightarrow[0,+\infty)$. Assume that $\Psi$ satisfies the Lipschitz condition with a Lipschitz constant $\lambda \in(0,+\infty)$, i.e., $d(\Psi(x), \Psi(y)) \leqslant \lambda d(x, y)$ for all $x, y \in Y$, and $H_{j}(x):=\sum_{i=0}^{\infty} \lambda^{i} h_{j}\left(\alpha^{i}(x)\right)$ is convergent for every $x \in K$ and $j=1,2$.

THEOREM 2.1. Let $f: K \rightarrow Y$ be a function such that

$$
\begin{array}{ll}
d(\Psi \circ f \circ \alpha(x), f(x)) \leqslant h_{1}(x), & x \in K, \\
d(f(x), \Psi \circ f \circ \alpha(x)) \leqslant h_{2}(x), & x \in K .
\end{array}
$$

Then the limit

$$
F(x):=\lim _{n \rightarrow \infty} \Psi^{n} \circ f \circ \alpha^{n}(x)
$$

exists for every $x \in K$ and the function $F: K \rightarrow Y$ is a unique solution of the equation $\Psi \circ F \circ \alpha=F$ such that

$$
\begin{aligned}
& d(F(x), f(x)) \leqslant H_{1}(x), \quad x \in K, \\
& d(f(x), F(x)) \leqslant H_{2}(x), \quad x \in K .
\end{aligned}
$$

Proof. We claim that for all $n \in \mathbb{N}$ the following two inequalities hold:

$$
\begin{array}{ll}
d\left(\Psi^{n} \circ f \circ \alpha^{n}(x), f(x)\right) \leqslant \sum_{i=0}^{n-1} \lambda^{i} h_{1}\left(\alpha^{i}(x)\right), & x \in K, \\
d\left(f(x), \Psi^{n} \circ f \circ \alpha^{n}(x)\right) \leqslant \sum_{i=0}^{n-1} \lambda^{i} h_{2}\left(\alpha^{i}(x)\right), & x \in K .
\end{array}
$$

For $n=1$ these inequalities hold by the assumption. Let $n \in \mathbb{N}$ and suppose that $(2.1)$ and $(2.2)$ are true. From the triangle inequality $(\mathrm{Q} 2)$, the Lipschitz condition, the assumption and the induction hypothesis we have

$$
\begin{aligned}
d\left(\Psi^{n+1} \circ f \circ \alpha^{n+1}(x), f(x)\right) \leqslant & d\left(\Psi^{n+1} \circ f \circ \alpha^{n+1}(x), \Psi \circ f \circ \alpha(x)\right) \\
& +d(\Psi \circ f \circ \alpha(x), f(x)) \\
\leqslant & \lambda d\left(\Psi^{n} \circ f \circ \alpha^{n}(\alpha(x)), f(\alpha(x))\right)+h_{1}(x)
\end{aligned}
$$




$$
\begin{aligned}
\leqslant \lambda \sum_{i=0}^{n-1} \lambda^{i} h_{1}\left(\alpha^{i}(\alpha(x))\right)+h_{1}(x) & =\sum_{i=0}^{n-1} \lambda^{i+1} h_{1}\left(\alpha^{i+1}(x)\right)+h_{1}(x) \\
& =\sum_{i=1}^{n} \lambda^{i} h_{1}\left(\alpha^{i}(x)\right)+h_{1}(x)=\sum_{i=0}^{n} \lambda^{i} h_{1}\left(\alpha^{i}(x)\right)
\end{aligned}
$$

for all $x \in K$. Analogously, we can prove that

$$
d\left(f(x), \Psi^{n+1} \circ f \circ \alpha^{n+1}(x)\right) \leqslant \sum_{i=0}^{n} \lambda^{i} h_{2}\left(\alpha^{i}(x)\right)
$$

for every $x \in K$, so the claim follows.

Now, we show that for all $m \in \mathbb{N}, k \in \mathbb{N} \cup\{0\}$ we have

(2.3) $d\left(\Psi^{m+k} \circ f \circ \alpha^{m+k}(x), \Psi^{k} \circ f \circ \alpha^{k}(x)\right) \leqslant \sum_{i=k}^{m+k-1} \lambda^{i} h_{1}\left(\alpha^{i}(x)\right), x \in K$,

(2.4) $d\left(\Psi^{k} \circ f \circ \alpha^{k}(x), \Psi^{m+k} \circ f \circ \alpha^{m+k}(x)\right) \leqslant \sum_{i=k}^{m+k-1} \lambda^{i} h_{2}\left(\alpha^{i}(x)\right), x \in K$.

Let $m \in \mathbb{N}$. For $k=0$ these conditions are simply the inequalities $\sqrt{2.1}$ and 2.2 with $n=m$. Thus, take any $k \in \mathbb{N}$ and suppose that (2.3) and (2.4) are true. Then

$$
\begin{aligned}
& d\left(\Psi^{m+k+1} \circ f \circ \alpha^{m+k+1}(x), \Psi^{k+1} \circ f \circ \alpha^{k+1}(x)\right) \\
& \leqslant \lambda d\left(\Psi^{m+k} \circ f \circ \alpha^{m+k}(\alpha(x)), \Psi^{k} \circ f \circ \alpha^{k}(\alpha(x))\right) \\
& \leqslant \lambda \sum_{i=k}^{m+k-1} \lambda^{i} h_{1}\left(\alpha^{i}(\alpha(x))\right) \\
& \quad=\sum_{i=k}^{m+k-1} \lambda^{i+1} h_{1}\left(\alpha^{i+1}(x)\right)=\sum_{i=k+1}^{m+k} \lambda^{i} h_{1}\left(\alpha^{i}(x)\right)
\end{aligned}
$$

for all $x \in K$. In the same way we can show that

$$
d\left(\Psi^{k+1} \circ f \circ \alpha^{k+1}(x), \Psi^{m+k+1} \circ f \circ \alpha^{m+k+1}(x)\right) \leqslant \sum_{i=k+1}^{m+k} \lambda^{i} h_{2}\left(\alpha^{i}(x)\right)
$$

for every $x \in K$. Therefore inequalities 2.3 and $(2.4)$ are proved. 
We conclude from this that for all $m \in \mathbb{N}, k \in \mathbb{N} \cup\{0\}$

$$
\begin{aligned}
& d\left(\Psi^{m+k} \circ f \circ \alpha^{m+k}(x), \Psi^{k} \circ f \circ \alpha^{k}(x)\right) \leqslant \sum_{i=k}^{\infty} \lambda^{i} h_{1}\left(\alpha^{i}(x)\right), \quad x \in K, \\
& d\left(\Psi^{k} \circ f \circ \alpha^{k}(x), \Psi^{m+k} \circ f \circ \alpha^{m+k}(x)\right) \leqslant \sum_{i=k}^{\infty} \lambda^{i} h_{2}\left(\alpha^{i}(x)\right), \quad x \in K .
\end{aligned}
$$

Consequently, for every $x \in K$ and for every $\varepsilon>0$ there exists $k \in \mathbb{N}$ such that for all $m, l \in \mathbb{N}$ we have

$$
\begin{aligned}
& d\left(\Psi^{m+k} \circ f \circ \alpha^{m+k}(x), \Psi^{l+k} \circ f \circ \alpha^{l+k}(x)\right) \\
& \leqslant d\left(\Psi^{m+k} \circ f \circ \alpha^{m+k}(x), \Psi^{k} \circ f \circ \alpha^{k}(x)\right) \\
&+d\left(\Psi^{k} \circ f \circ \alpha^{k}(x), \Psi^{l+k} \circ f \circ \alpha^{l+k}(x)\right) \\
& \leqslant \sum_{i=k}^{\infty} \lambda^{i} h_{1}\left(\alpha^{i}(x)\right)+\sum_{i=k}^{\infty} \lambda^{i} h_{2}\left(\alpha^{i}(x)\right)<\varepsilon .
\end{aligned}
$$

It means that $\left(\Psi^{n} \circ f \circ \alpha^{n}(x)\right)_{n=1}^{\infty}$ is a Cauchy sequence for every $x \in K$. The space $(Y, d)$ is complete, so the limit $F(x):=\lim _{n \rightarrow \infty} \Psi^{n} \circ f \circ \alpha^{n}(x)$ exists for every $x \in K$.

Observe that

$$
\begin{aligned}
d(F(x), f(x)) & \leqslant d\left(F(x), \Psi^{n} \circ f \circ \alpha^{n}(x)\right)+d\left(\Psi^{n} \circ f \circ \alpha^{n}(x), f(x)\right) \\
& \leqslant d\left(F(x), \Psi^{n} \circ f \circ \alpha^{n}(x)\right)+H_{1}(x)
\end{aligned}
$$

for $x \in K, n \in \mathbb{N}$, what implies $d(F(x), f(x)) \leqslant H_{1}(x)$ for all $x \in K$. Similarly, we can show that $d(f(x), F(x)) \leqslant H_{2}(x)$ for every $x \in K$. Furthermore, for every $x \in K$ we have

$$
\begin{aligned}
0 & \leqslant d(\Psi \circ F \circ \alpha(x), F(x)) \\
& \leqslant d\left(\Psi(F(\alpha(x))), \Psi^{n+1} \circ f \circ \alpha^{n+1}(x)\right)+d\left(\Psi^{n+1} \circ f \circ \alpha^{n+1}(x), F(x)\right) \\
& \leqslant \lambda d\left(F(\alpha(x)), \Psi^{n} \circ f \circ \alpha^{n}(\alpha(x))\right)+d\left(\Psi^{n+1} \circ f \circ \alpha^{n+1}(x), F(x)\right),
\end{aligned}
$$

thus, $d(\Psi \circ F \circ \alpha(x), F(x))=0$ for $x \in K$. Similarly, $d(F(x), \Psi \circ F \circ \alpha(x))=0$ for $x \in K$. This implies that $\Psi \circ F \circ \alpha=F$. 
Suppose that $G: K \rightarrow Y$ is a function such that $\Psi \circ G \circ \alpha=G$ and

$$
\begin{aligned}
& d(G(x), f(x)) \leqslant H_{1}(x), \quad x \in K, \\
& d(f(x), G(x)) \leqslant H_{2}(x), \quad x \in K .
\end{aligned}
$$

By induction we obtain $\Psi^{n} \circ G \circ \alpha^{n}=G$ and $\Psi^{n} \circ F \circ \alpha^{n}=F$ for all $n \in \mathbb{N}$, so

$$
\begin{aligned}
0 \leqslant d(F(x), G(x)) & =d\left(\Psi^{n} \circ F \circ \alpha^{n}(x), \Psi^{n} \circ G \circ \alpha^{n}(x)\right) \\
& \leqslant \lambda^{n} d\left(F\left(\alpha^{n}(x)\right), G\left(\alpha^{n}(x)\right)\right) \\
& \leqslant \lambda^{n}\left[d\left(F\left(\alpha^{n}(x)\right), f\left(\alpha^{n}(x)\right)\right)+d\left(f\left(\alpha^{n}(x)\right), G\left(\alpha^{n}(x)\right)\right)\right] \\
& \leqslant \lambda^{n} \sum_{i=0}^{\infty} \lambda^{i} h_{1}\left(\alpha^{i+n}(x)\right)+\lambda^{n} \sum_{i=0}^{\infty} \lambda^{i} h_{2}\left(\alpha^{i+n}(x)\right) \\
& =\sum_{i=0}^{\infty} \lambda^{i+n} h_{1}\left(\alpha^{i+n}(x)\right)+\sum_{i=0}^{\infty} \lambda^{i+n} h_{2}\left(\alpha^{i+n}(x)\right) \\
& =\sum_{i=n}^{\infty} \lambda^{i} h_{1}\left(\alpha^{i}(x)\right)+\sum_{i=n}^{\infty} \lambda^{i} h_{2}\left(\alpha^{i}(x)\right)
\end{aligned}
$$

for all $x \in K$ and $n \in \mathbb{N}$. This implies that $d(F(x), G(x))=0$ for $x \in K$. In the same way we obtain $d(G(x), F(x))=0$ for $x \in K$, so $F=G$ and the theorem follows.

\section{Applications}

If $(G,+)$ is a groupoid (i.e., $G$ is a nonempty set and $+: G^{2} \rightarrow G$ is a binary operation, not necessarily commutative), then we say that it is uniquely divisible by 2 if for every $x \in G$ there exists a unique $y \in G$ such that $x=y+y=: 2 y$. In this case $y$ is denoted by $\frac{1}{2} x$ or $\frac{x}{2}$. A groupoid $(G,+)$ is called square symmetric iff $2(x+y)=2 x+2 y$ for all $x, y \in G$.

Consider the following notations: $2^{0} x=x, 2^{n+1} x=2\left(2^{n} x\right)$ for $x \in G$ and $n \in \mathbb{N}$. Moreover, if $(G,+)$ is uniquely divisible by 2 we write $2^{-1} x=\frac{1}{2} x$, $2^{-n-1} x=2^{-1}\left(2^{-n} x\right)$ for $x \in G, n \in \mathbb{N}$.

Let $(X,+),(Y,+)$ be square symmetric groupoids. Assume that $(X,+)$ is uniquely divisible by $2,(Y, d)$ is a complete dq-metric space and the operation $+: Y \times Y \rightarrow Y$ is continuous, i.e., if $x_{n} \rightarrow x$ and $y_{n} \rightarrow y$ then $x_{n}+y_{n} \rightarrow x+y$ 
for all sequences $\left(x_{n}\right)_{n=1}^{\infty},\left(y_{n}\right)_{n=1}^{\infty}$ of elements of the space $Y$ and $x, y \in Y$. Consider also a nonempty set $K \subset X$ and functions $\chi_{i}: K \times K \rightarrow[0, \infty)$, $i=1,2$.

With this notations we have the following corollary from Theorem 2.1.

Corollary 3.1. Suppose that $\frac{1}{2} K \subset K$ and that there exist constants $\eta_{1}, \eta_{2}, \varepsilon \in(0, \infty)$ such that $\eta_{i} \varepsilon<1$ and

$$
\begin{gathered}
\chi_{i}\left(\frac{x}{2}, \frac{y}{2}\right) \leqslant \eta_{i} \chi_{i}(x, y), \quad x, y \in K, i=1,2, \\
d(2 x, 2 y) \leqslant \varepsilon d(x, y), \quad x, y \in Y .
\end{gathered}
$$

Let $\varphi: K \rightarrow Y$ be a function that satisfies the conditions:

$$
\begin{gathered}
d(\varphi(x)+\varphi(y), \varphi(x+y)) \leqslant \chi_{1}(x, y), \\
d(\varphi(x+y), \varphi(x)+\varphi(y)) \leqslant \chi_{2}(x, y)
\end{gathered}
$$

for all $x, y \in K, x+y \in K$. Then there exists a unique solution $F: K \rightarrow Y$ of the equation

$$
F(x+y)=F(x)+F(y), \quad x, y \in K, x+y \in K
$$

such that

$$
d(F(x), \varphi(x)) \leqslant \frac{\eta_{1} \chi_{1}(x, x)}{1-\eta_{1} \varepsilon}, \quad x \in K
$$

and

$$
d(\varphi(x), F(x)) \leqslant \frac{\eta_{2} \chi_{2}(x, x)}{1-\eta_{2} \varepsilon}, \quad x \in K .
$$

Proof. By the assumption, for every $x \in K$,

$$
d\left(2 \varphi\left(\frac{x}{2}\right), \varphi(x)\right)=d\left(\varphi\left(\frac{x}{2}\right)+\varphi\left(\frac{x}{2}\right), \varphi\left(\frac{x}{2}+\frac{x}{2}\right)\right) \leqslant \chi_{1}\left(\frac{x}{2}, \frac{x}{2}\right)
$$

and similarly

$$
d\left(\varphi(x), 2 \varphi\left(\frac{x}{2}\right)\right) \leqslant \chi_{2}\left(\frac{x}{2}, \frac{x}{2}\right), x \in K .
$$

Setting $f=\varphi, \Psi(x)=2 x, \lambda=\varepsilon, h_{i}(x)=\chi_{i}\left(\frac{x}{2}, \frac{x}{2}\right), i=1,2$, and $\alpha(x)=\frac{x}{2}$ in Theorem 2.1. the limit $F(x)=\lim _{n \rightarrow \infty} 2^{n} \varphi\left(2^{-n} x\right)$ exists for every $x \in K$ and 
it is a unique solution of the equation $2 F\left(2^{-1} x\right)=F(x), x \in K$, such that for every $x \in K$,

$$
\begin{aligned}
d(F(x), \varphi(x)) & \leqslant \sum_{i=0}^{\infty} \varepsilon^{i} \chi_{1}\left(2^{-i} \frac{x}{2}, 2^{-i} \frac{x}{2}\right) \leqslant \sum_{i=0}^{\infty} \varepsilon^{i} \eta_{1}^{i} \chi_{1}\left(\frac{x}{2}, \frac{x}{2}\right) \\
& \leqslant \eta_{1} \chi_{1}(x, x) \sum_{i=0}^{\infty}\left(\varepsilon \eta_{1}\right)^{i}=\frac{\eta_{1} \chi_{1}(x, x)}{1-\eta_{1} \varepsilon}
\end{aligned}
$$

and analogously,

$$
d(\varphi(x), F(x)) \leqslant \frac{\eta_{2} \chi_{2}(x, x)}{1-\eta_{2} \varepsilon}, \quad x \in K .
$$

Let $x, y \in K, x+y \in K$. Observe that for all $n \in \mathbb{N}$ we have

$$
\begin{aligned}
d\left(2^{n} \varphi\left(2^{-n} x\right)+2^{n} \varphi\left(2^{-n} y\right),\right. & \left.2^{n} \varphi\left(2^{-n}(x+y)\right)\right) \\
& \leqslant \varepsilon^{n} d\left(\varphi\left(2^{-n} x\right)+\varphi\left(2^{-n} y\right), \varphi\left(2^{-n}(x+y)\right)\right) \\
& \leqslant \varepsilon^{n} \chi_{1}\left(2^{-n} x, 2^{-n} y\right) \leqslant\left(\varepsilon \eta_{1}\right)^{n} \chi_{1}(x, y) .
\end{aligned}
$$

Thus,

$$
\lim _{n \rightarrow \infty} d\left(2^{n} \varphi\left(2^{-n} x\right)+2^{n} \varphi\left(2^{-n} y\right), 2^{n} \varphi\left(2^{-n}(x+y)\right)\right)=0 .
$$

Similarly,

$$
\lim _{n \rightarrow \infty} d\left(2^{n} \varphi\left(2^{-n}(x+y)\right), 2^{n} \varphi\left(2^{-n} x\right)+2^{n} \varphi\left(2^{-n} y\right)\right)=0 .
$$

For all $n \in \mathbb{N}$

$$
\begin{aligned}
0 \leqslant & d(F(x)+F(y), F(x+y)) \\
\leqslant & d\left(F(x)+F(y), 2^{n} \varphi\left(2^{-n} x\right)+2^{n} \varphi\left(2^{-n} y\right)\right) \\
& +d\left(2^{n} \varphi\left(2^{-n} x\right)+2^{n} \varphi\left(2^{-n} y\right), 2^{n} \varphi\left(2^{-n}(x+y)\right)\right) \\
& +d\left(2^{n} \varphi\left(2^{-n}(x+y)\right), F(x+y)\right),
\end{aligned}
$$

what implies $d(F(x)+F(y), F(x+y))=0$. In the same way we can prove that $d(F(x+y), F(x)+F(y))=0$, hence $F(x+y)=F(x)+F(y)$. 
Suppose that a function $G: K \rightarrow Y$ satisfies the conditions:

$$
\begin{gathered}
G(x+y)=G(x)+G(y), \quad x, y \in K, x+y \in K, \\
d(G(x), \varphi(x)) \leqslant \frac{\eta_{1} \chi_{1}(x, x)}{1-\eta_{1} \varepsilon}, \quad x \in K, \\
d(\varphi(x), G(x)) \leqslant \frac{\eta_{2} \chi_{2}(x, x)}{1-\eta_{2} \varepsilon}, \quad x \in K .
\end{gathered}
$$

Then

$$
2 G\left(\frac{x}{2}\right)=G\left(\frac{x}{2}\right)+G\left(\frac{x}{2}\right)=G\left(\frac{x}{2}+\frac{x}{2}\right)=G(x)
$$

for every $x \in K$, so by the uniqueness in Theorem 2.1 we obtain $F=G$.

Assume that $(X,+)$ and $(Y,+)$ are abelian groups, $c \in X, C \in Y$. Let $a, b: X \rightarrow X, A, B: Y \rightarrow Y$ be homomorphisms such that $a \circ b=b \circ a$ and $A \circ B=B \circ A$. Suppose that the function $\alpha: X \ni x \longmapsto a(x)+b(x)+c \in$ $X$ is bijective and $(Y, d)$ is a complete dq-metric space. Assume also that $x_{n} \rightarrow x$ and $y_{n} \rightarrow y$ implies $A\left(x_{n}\right)+B\left(y_{n}\right)+C \rightarrow A(x)+B(y)+C$ for all sequences $\left(x_{n}\right)_{n=1}^{\infty},\left(y_{n}\right)_{n=1}^{\infty}$ of elements of the space $Y$ and $x, y \in Y$ and consider a nonempty subset $K \subset X$ and functions $\chi_{i}: K \times K \rightarrow[0, \infty)$, $i=1,2$. The next corollary corresponds in particular to the recent results in [2, 7, 8].

Corollary 3.2. Suppose that $\alpha^{-1}(K) \subset K$ and that there exist constants $\eta_{1}, \eta_{2}, \varepsilon \in(0, \infty)$ such that $\eta_{i} \varepsilon<1$ and

$$
\begin{gathered}
\chi_{i}(x, y) \leqslant \eta_{i} \chi_{i}(a(x)+b(x)+c, a(y)+b(y)+c), \quad x, y \in K, i=1,2, \\
d(A(x)+B(x)+C, A(y)+B(y)+C) \leqslant \varepsilon d(x, y), \quad x, y \in Y .
\end{gathered}
$$

Let $\varphi: K \rightarrow Y$ be a function that satisfies the conditions:

$$
\begin{aligned}
& d(A(\varphi(x))+B(\varphi(y))+C, \varphi(a(x)+b(y)+c)) \leqslant \chi_{1}(x, y) \\
& d(\varphi(a(x)+b(y)+c), A(\varphi(x))+B(\varphi(y))+C) \leqslant \chi_{2}(x, y)
\end{aligned}
$$

for all $x, y \in K, a(x)+b(y)+c \in K$. Then there exists a unique solution $F: K \rightarrow Y$ of equation

$$
F(a(x)+b(y)+c)=A(F(x))+B(F(y))+C, \quad x, y \in K, a(x)+b(y)+c \in K
$$


such that

$$
\begin{array}{ll}
d(F(x), \varphi(x)) \leqslant \frac{\eta_{1} \chi_{1}(x, x)}{1-\eta_{1} \varepsilon}, & x \in K, \\
d(\varphi(x), F(x)) \leqslant \frac{\eta_{2} \chi_{2}(x, x)}{1-\eta_{2} \varepsilon}, & x \in K .
\end{array}
$$

Proof. Define operations $\star: X \times X \rightarrow X$ and $\diamond: Y \times Y \rightarrow Y$ by:

$$
\begin{gathered}
x \star y=a(x)+b(y)+c, \quad x, y \in X, \\
z \diamond w=A(z)+B(w)+C, \quad z, w \in Y .
\end{gathered}
$$

Then $(X, \star)$ and $(Y, \diamond)$ form groupoids that are a square symmetric, because $a, b$ and $A, B$ are homomorphisms such that $a \circ b=b \circ a$ and $A \circ B=B \circ A$. Moreover, since the function $\alpha$ is bijective, $(X, \star)$ is uniquely divisible by 2 . Note that $2 x=x \star x=\alpha(x)$ for $x \in X$ and $2 z=z \diamond z=A(z)+B(z)+C$ for $z \in Y$, so 3.1 and 3.2 take the forms

$$
\begin{gathered}
\chi_{i}(x, y) \leqslant \eta_{i} \chi_{i}(2 x, 2 y), \quad x, y \in K, i=1,2, \\
d(2 x, 2 y) \leqslant \varepsilon d(x, y), \quad x, y \in Y
\end{gathered}
$$

and $(3.3)$ and $(3.4)$ can be written as

$$
\begin{aligned}
& d(\varphi(x) \diamond \varphi(y), \varphi(x \star y)) \leqslant \chi_{1}(x, y) \\
& d(\varphi(x \star y), \varphi(x) \diamond \varphi(y)) \leqslant \chi_{2}(x, y)
\end{aligned}
$$

for all $x, y \in K, x \star y \in K$. From these observations and from Corollary 3.1 the statement follows.

Acknowledgements. The author would like to thank Professor Janusz Brzdęk for useful comments and helpful suggestions concerning this paper.

\section{References}

[1] Amini-Harandi A., Metric-like spaces, partial metric spaces and fixed points, Fixed Point Theory Appl. 2012, 2012:204, 10 pp.

[2] Bahyrycz A., Piszczek M., Hyperstability of the Jensen functional equation, Acta Math. Hungar. 142 (2014), 353-365. 
[3] Brillouët-Belluot N., Brzdęk J., Ciepliński K., On some recent developments in Ulam's type stability, Abstr. Appl. Anal. 2012, Art. ID 716936, 41 pp.

[4] Brzdęk J., On a method of proving the Hyers-Ulam stability of functional equations on restricted domains, Aust. J. Math. Anal. Appl. 6 (2009), Art. 4, 10 pp.

[5] Hyers D.H., On the stability of the linear functional equation, Proc. Nat. Acad. Sci. U.S.A. $27(1 \overline{941}), 222-224$.

[6] Hyers D.H., Isac G., Rassias T.M., Stability of Functional Equations in Several Variables, Birkhäuser Boston, Boston, 1998.

[7] Piszczek M., Remark on hyperstability of the general linear equation, Aequationes Math. 88 (2014), 163-168.

[8] Piszczek M., Hyperstability of the general linear functional equation, Bull. Korean Math. Soc. 52 (2015), 1827-1838.

[9] Rahman M.U., Sarwar M., Some new fixed point theorems in dislocated quasi-metric spaces, Palest. J. Math. 5 (2016), 171-176.

[10] Sarwar M., Rahman M.U., Ali G., Some fixed point results in dislocated quasi-metric (dq-metric) spaces, J. Inequal. Appl. 2014, 2014:278, 11 pp.

[11] Ulam S.M., A Collection of Mathematical Problems, Interscience Publishers, New York-London, 1960. Reprinted as: Problems in Modern Mathematics, John Wiley \& Sons, New York, 1964.

Department of Mathematics

Pedagogical University of Cracow

PODCHORĄŻYCH 2

30-084 KraKóW

PolAnd

e-mail: bhejmej1f@gmail.com 